\title{
Relationship of induced motion and apparent straight-ahead shifts to optokinetic stimulus velocity
}

\author{
ROBERT B. POST and LORI A. LOTT \\ University of California, Davis, California
}

\begin{abstract}
Induced motion (IM) of a fixated spot stimulus and shifts of the apparent straight-ahead (ASA) from the objective median plane were studied as a function of the velocity of a full-field optokinetic background stimulus. Both IM and ASA were influenced similarly by changes in stimulus velocity. The magnitude of both responses, averaged across subjects, increased to a peak level with background velocities of $40-80 \mathrm{deg} / \mathrm{sec}$ and decreased at higher velocities. Individual subjects differed with respect to the precise functions by which IM and ASA shifts were related to stimulus velocity. However, for individual subjects, the effects of velocity on IM and ASA shifts were typically highly correlated. Although IM is correlated with shifts of ASA in the opposite direction, the magnitude of the ASA shift is insufficient to account for the observed IM.
\end{abstract}

Induced motion (IM), the illusory motion of a visual stimulus opposite to the real motion of other stimuli, has been attributed to a variety of causes. Early work by Duncker (1929), as well as several recent investigators (e.g., Mack, Heuer, Fendrich, Vilardi, \& Chambers, 1985; Wallach, Bacon, \& Schulman, 1978), emphasized the role, in motion perception, of the relative localization of the elements in the visual display. These accounts of IM are at times referred to as "object-relative," because they suggest that illusory motion results from change in the perceived location of one object relative to the perceived location of another.

Other theories of IM emphasize the role of a variety of factors associated with either the perceived spatial coordinates or the spatial orientation responses of the subject viewing the IM display. Among these is the hypothesis that the inducing stimulus causes the subject's perception of straight-ahead, or apparent straight-ahead (ASA), to be shifted in the direction of inducer motion. This, in turn, results in the perception that the stimulus that has motion induced in it is moving in the direction opposite that of ASA (Bridgeman \& Klassen, 1983; Brosgole, 1968).

A second hypothesis that implicates a role of spatial orientation responses in the genesis of IM is that the inducing stimulus (particularly if it is large) produces a perception of self-motion (vection) of the subject in the direction opposite to that of the inducer motion. Any visual detail not moving with the inducer is therefore seen as moving with the observer in the opposite direction (Howard, 1989; Johansson, 1977).

This research was supported in part by University of California Grant D-1832 to R.B.P. The assistance of Jessica Chen in data reduction and analysis is gratefully acknowledged. The help of Amy Exelby in the collection of data is also appreciated. Correspondence may be addressed to either author at the Department of Psychology, University of California, Davis, CA 95616.
A third factor associated with orientation responses and hypothesized to contribute to IM is the reflexive optokinetic nystagmus (OKN), which would be elicited in the absence of a fixation stimulus. OKN represents the summed activity of two oculomotor subsystems termed "'indirect' and 'direct"' OKN (Cohen, Matsuo, \& Raphan, 1977; see Collewijn, 1985, for a review). The indirect component, which is phylogenetically older, evolved in the interest of maintaining gaze stability during self-motion. The relatively newer direct OKN system, which subserves the pursuit of small moving objects against stationary backgrounds, may also either oppose or supplement activity in the indirect system. Post and Leibowitz (1985) have proposed that when OKN is suppressed by fixation of a visual stimulus, activity in the direct system results in the perception of motion in the fixated stimulus in the direction opposite to that of optokinetic stimulation, hence IM (e.g., Heckmann \& Post, 1988; Post, 1986).

Although each of these three hypotheses regarding spatial orientation responses proposes a distinct mechanism for the genesis of IM, there is evidence that they are related, in that each represents a visual-vestibular interaction. That is, ASA shifts, vection, and indirect OKN all result from activation of the vestibular system by visual stimulation (for a review, see Dichgans \& Brandt, 1978). Therefore, it might be expected that visual stimuli that are effective in eliciting vestibular activation would be particularly effective stimuli for eliciting IM. The finding that one of these responses may be found under conditions that give rise to IM does not by itself ensure that the observed IM is actually the result of that mechanism. Rather, it might result from one or both of the other proposed mediating responses, which would also tend to be activated at the same time. Because IM may be elicited by a wide range of sizes of inducing stimuli (for a review, see 
Reinhardt-Rutland, 1988), it is unlikely that each of the proposed visual-vestibular causes of IM operate under all conditions in which IM is observed. Specifically, with small inducing stimuli, which are incapable of eliciting vection, it is unlikely that vection sensations contribute to IM. Under the same conditions, however, ASA shifts and $\mathrm{OKN}$ suppression would still be operative.

The present paper concerns the relationship of IM and ASA shifts to optokinetic stimulus velocity. In previous studies, it has been demonstrated that displacement of a visual stimulus produces an ASA shift in the direction of stimulus motion, and correlated IM of a stationary stimulus in the opposite direction, leading to the hypothesis that the ASA shift causes IM (Bridgeman \& Klassen, 1983; Brosgole, 1968). Post and Heckmann (1986) also found that ASA shifts were paired with opposite IM, and that the magnitude of both was correlated as a function of the duration of the inducing stimulus. These authors questioned, however, whether the ASA shifts produce IM, because the velocity of IM was correlated with the spatial extent of ASA displacement, and not with the velocity of ASA motion. At longer durations, the greatest IM velocities were obtained, but the ASA was stationary at a large displacement from objective straight-ahead. Therefore, the ASA shifts were not sufficient to produce the observed IM. Mack et al. (1985) likewise observed ASA shifts under conditions that produced opposite IM, but that were of insufficient magnitude to have produced the IM.

The research in the present paper was carried out to examine further the relationship of ASA shifts to IM. Both responses were measured as the velocity of the inducing stimulus was changed, to determine whether or not they would remain correlated across changes in this variable in a manner similar to that reported previously for changes in stimulus duration (Post \& Heckmann, 1986). To avoid the confounding of stimulus velocity with duration of stimulus motion, a large, repetitive stripe pattern that moved in only one direction on any given trial was used rather than an oscillating inducer stimulus.

\section{Method}

Seven individuals, 20 to 30 years old, served as subjects. Six were naive with respect to the experimental hypothesis. The seventh was one of the authors (L.L.). Each was emmetropic or mildly myopic, and did not experience difficulty in focusing at the viewing distance of the display.

The apparatus has been described in detail previously (Post \& Heckmann, 1986). Observations were made with the subjects seated, and head position and viewing distance were maintained with a chinrest. All observations were binocular. The inducer stimulus consisted of a full field of long vertical black and white stripes that moved either leftward or rightward. These were formed by rotating a striped cylindrical shadow caster around an incandescent light source centered above the subject's head. The shadows appeared on a Plexiglas hemicylinder concentric with the shadow caster. Viewing distance was $50 \mathrm{~cm}$. Each black-white pair subtended $15 \mathrm{deg}$, with luminances for the white and black portions of 1.13 and $.06 \mathrm{~cd} / \mathrm{m}^{2}$, respectively. The contrast was $87 \%$.

The fixation stimulus consisted of a spot of light from a laser (HeNe) reflected off of a mirror attached to a galvanometer and function generator, so that the laser spot moved repeatedly upward through an arc of $14.5 \mathrm{deg}$ at a rate of $7.1 \mathrm{deg} / \mathrm{sec}$. This real vertical motion of the spot combines with an illusory horizontal component (IM) to yield apparent motion on a slant. The slant of the apparent motion path indicates the magnitude of the horizontal IM component (Wallach et al., 1978). The only other visual detail was a horizontal panel located in the lower visual field, which occluded from view portions of the apparatus that would otherwise provide reference detail concerning the horizontal location of stimuli.

Subjects initially sat with eyes closed and started each experimental trial by pressing a button, simultaneously opening their eyes and fixating the upward moving fixation spot. A rod grasped with the left hand was adjusted continuously so that its slant matched the apparent slope of the motion path of the tracked stimulus. The rod was connected to a potentiometer, and a microcomputer sampled the voltage variations associated with changes in the orientation of the rod. These were converted into the horizontal velocity of IM and displayed on the computer terminal. The IM velocity displayed at the end of $30 \mathrm{sec}$ was recorded as the IM measure. At this time, the subject indicated the location of ASA by reaching out with an index finger and touching the surface of the screen. At the moment the screen was touched, the subject shut his or her eyes, and the horizontal distance between the fingertip and moving spot was recorded by the experimenter as the corresponding ASA measure.

Measures were obtained with velocities of $0,10,20,40,80$ and $160 \mathrm{deg} / \mathrm{sec}$ for the moving stripes. Four experimental sessions, each consisting of a total of 12 trials, were run for each subject for both directions of background motion. To minimize the likelihood of adaptation effects between trials (Wallach et al., 1978), $30 \mathrm{sec}$ of rest with eyes shut preceded each trial, and a break of 3 min separated the first and second group of 6 trials. Within each group of 6 trials, each velocity was tested once, and the order of velocities was counterbalanced.

\section{RESULTS}

The slant judgments and pointing responses of the subjects indicated that the moving stripes created both IM of the fixation stimulus and shifts of the ASA. For each of the subjects, the spot was seen to move in the direction opposite that of stripe motion on each of the trials at nonzero velocities. Similarly, ASA was shifted from the objective median plane in the direction of the stripe motion on $94.6 \%$ of all trials with nonzero velocities.

The velocity of IM typically increased during the 30sec exposure period, in a manner similar to that reported previously (Heckmann \& Post, 1988; Post \& Heckmann, 1986). An example of the time course of this increase for velocities of 10 and $40 \mathrm{deg} / \mathrm{sec}$ is shown in Figure 1. Comparison of the data in this figure with the ASA shifts obtained for this subject at these velocities also supports the previously reported finding (Mack et al., 1985) that the magnitude of ASA shifts is too small to produce the total IM measured. Specifically, this subject's ASA shifts at the end of $30 \mathrm{sec}$ averaged 5.2 and $9.4 \mathrm{deg}$ for $10-$ and 40- deg/sec stimulation, respectively. However, integration under the traces in Figure 1 yields the total IM experienced as being 53 and $143 \mathrm{deg}$ for $10-$ and $40-\mathrm{deg} / \mathrm{sec}$ stimulation, respectively.

The mean IM and ASA shift values for all subjects were computed separately for each stimulus velocity and are presented in Figure 2. The magnitude of each response 


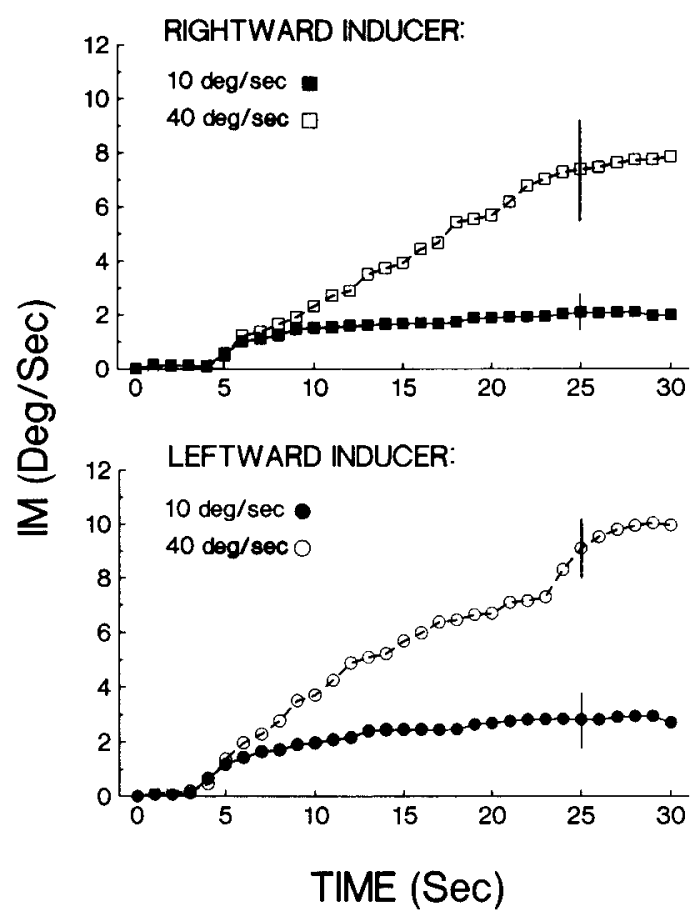

Figure 1. Induced-motion velocity as a function of stimulus duration for a naive subject at two optokinetic stimulus velocities. Plotted values indicate the mean of eight trials. Error bars represent plus or minus the mean of standard deviations associated with each point. increases with greater stimulus velocity to a peak at $40 \mathrm{deg} / \mathrm{sec}$, then decreases at higher velocities. The two responses are therefore highly symmetrical; large values of IM are accompanied by large ASA shifts in the opposite direction.

Separate analyses of variance were performed on the IM and ASA results presented in Figure 2. For the IM data, a significant main effect was obtained for the variable of stimulus velocity $[F(5,30)=9.81, p<.0001]$. Post hoc analyses determined that the differences between results obtained with $20-$ or $160-\mathrm{deg} / \mathrm{sec}$ stimulation and baseline were significant $(p<.01)$ and that the differences between 40 - or $80-\mathrm{deg} / \mathrm{sec}$ results and baseline were highly significant $(p<.001)$. Pairwise comparisons among nonzero velocity results indicated that IM was significantly greater with $40-$ or $80-\mathrm{deg} / \mathrm{sec}$ stimulation than with $10-\mathrm{deg} / \mathrm{sec}$ stimulation $(p<.05)$.

A similar pattern of results was obtained with the analysis of variance performed on the ASA results. A significant main effect was obtained for the variable of stimulus velocity $[F(5,30)=11.83, p<.0001]$. Post hoc analyses determined that the difference between results obtained with $10-$ or $160-\mathrm{deg} / \mathrm{sec}$ and $0-\mathrm{deg} / \mathrm{sec}$ (baseline) stimulation were significant $(p<.01)$ and that the differences between $20-, 40-$, or $80-\mathrm{deg} / \mathrm{sec}$ results and baseline were highly significant $(p<.001)$.

To demonstrate the relative effectiveness of different velocities in eliciting IM and ASA shifts, the mean IM and ASA values presented in Figure 2 were transformed
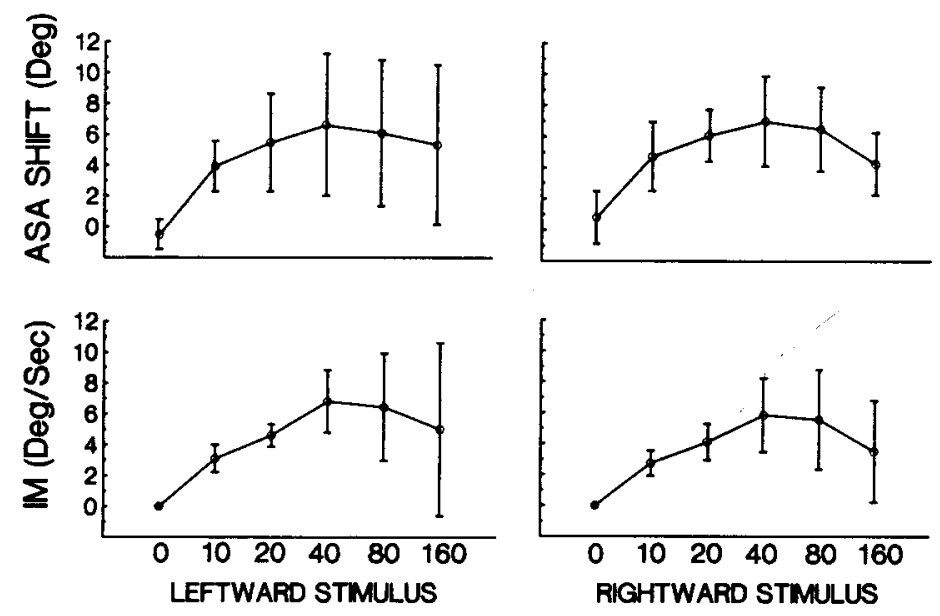

\section{STIMULUS VELOCITY (Deg/Sec)}

Figure 2. Induced-motion and apparent-straight-ahead shifts for all subjects at each optokinetic stimulus velocity. Error bars represent plus or minus one standard deviation about the plotted means. 
by dividing them by the velocity of the inducing stimulus. In the case of IM, this yields the gain of the response, with a value of 1.0 corresponding to IM equal in velocity to the inducing stimulus. In the case of ASA shifts, the transformation does not yield gain, since displacement is being divided by velocity; but the transformation does provide an analogous statistic that may be used for demonstrating the relative effectiveness of different optokinetic stimulus velocities. The transformed values which are presented in Figure 3 illustrate that with increases in velocity, the inducer stimulus becomes relatively less effective in eliciting both IM and ASA shifts. The observed decrease in the plotted values as a function of velocity is roughly exponential. As noted previously, the two responses are similarly influenced by changes in the velocity of the optokinetic stimulus.

The symmetry of IM and ASA responses is also apparent when the data of individual subjects are examined. Figure 4 permits visual comparison of IM magnitude and ASA shifts for 2 naive subjects. Although the overall functions obtained differ between subjects, the effect of stimulus velocity is highly similar for both IM magnitude and ASA shifts within subjects. Both subjects show a similar initial increase in IM and ASA shifts with higher stimulus velocities. However, these responses peak at $40 \mathrm{deg} / \mathrm{sec}$ for Subject A.E., whereas for Subject M.C., they tend to level out at higher rightward velocities and continue to increase with greater stimulus velocities in the leftward direction. Such individual differences in response patterns account for much of the variability present at higher stimulus velocities in the combined subjects' data in Figure 2.

Owing to the presence of individual differences, linear regression analyses of the relationship between the two response measures were performed separately for each subject. For 6 of the 7 subjects, the correlation between the two responses was statistically significant. Obtained coefficients of regression $r(46)$ ranged from .24 to .84 , $p$ s $<.05$ to .001 , respectively. For a 7 th subject, the correlation coefficient was not significant when all data were included in the analysis. When separate analyses were performed for each direction of inducer motion for this subject, a significant correlation between IM and ASA results was found for the data obtained with leftward stimulation $[r(22)=.46, p<.05]$.

\section{DISCUSSION}

Our primary finding in this study is that stimulus velocity exerts a highly similar influence on both IM and ASA responses. Both tend to be maximal with optokinetic stimulation in the vicinity of $40-80 \mathrm{deg} / \mathrm{sec}$, and then decrease at higher velocities. Although IM and ASA values are correlated, the magnitude of the ASA shifts is insufficient to produce the observed IM. Expression of IM and ASA measures relative to the velocity of the inducing stimulus demonstrates that the gain of both responses decreases as velocity increases.

The results of this study help clarify the effect of velocity on IM. Previous reports (Becklen \& Wallach, 1985; Wallach \& Becklin, 1983) have suggested that IM elicited with a large inducing stimulus declines with increased inducer velocity. This interpretation was questioned by Post, Chi, Heckmann, and Chaderjian (1989), who noted that an oscillating inducing stimulus had been used in such studies, thereby confounding the variable of stimulus velocity with duration of stimulus motion. In the present experiment, the optokinetic inducing stimulus did not oscillate, so the effects of velocity were examined independently of duration. With the factor of stimulus duration controlled, the present study demonstrates that IM per-
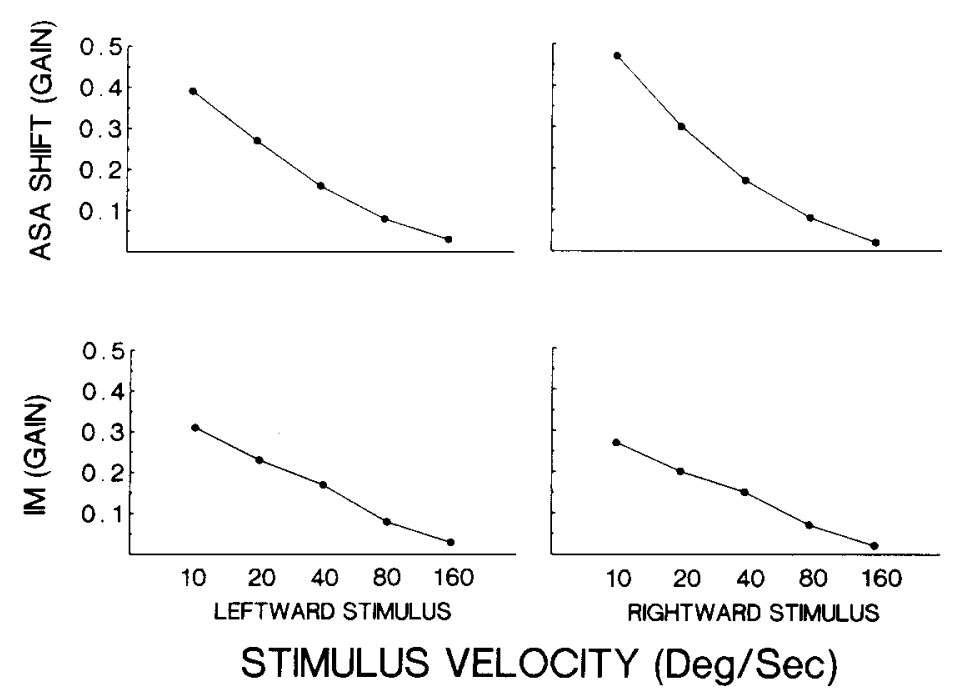

Figure 3. Data from Figure 2 transposed to express the magnitude of inducedmotion and apparent-straight-ahead shifts as a proportion of the velocity of the optokinetic stimulus. 


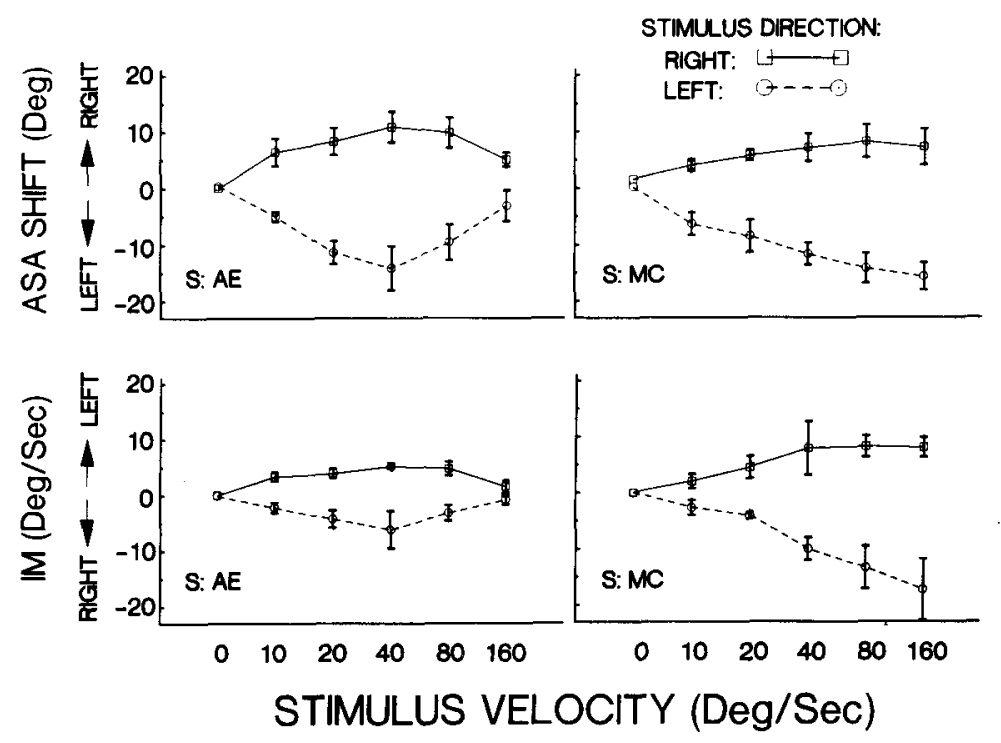

Figure 4. Induced-motion (IM) and apparent-straight-ahead (ASA) shifts as a function of optokinetic stimulus velocity for 2 naive subjects. To facilitate comparison of the two responses, IM and ASA measures obtained with rightward inducing stimuli are plotted as positive values, and the results for leftward stimulation are plotted as negative values. Error bars correspond to plus or minus one standard deviation about the plotted means.

sists at high inducer velocities, although the gain of the response decreases as stimulus velocity increases. In studies done with small inducing stimuli (e.g., Rock, Auster, Schiffman, \& Wheeler, 1980), a decline of IM has been reported for lower velocities than those reported in the present paper. It therefore appears likely that the influence of velocity on IM varies with the size of the inducing stimulus.

The data also provide new information about the effect of stimulus velocity on displacement of ASA. In a previous report (Brecher, Brecher, Kommerell, Sauter, \& Sellerbeck, 1972), in which the data from 1 subject were presented, ASA shifts elicited by a similar rotating surround stimulus increased as stimulus velocity increased up to about $100 \mathrm{deg} / \mathrm{sec}$, and decreased as stimulus velocity increased further. The present data are consistent with this pattern, but they also demonstrate the existence of individual differences.

Harris (1974) has argued that some ASA shifts are "cognitive or conceptual" in nature, and represent judgmental processes in subjects. These are distinct from "perceptual" ASA shifts, which are true shifts of subjective coordinates. The present experiment, through the use of a repetitive, striped inducing stimulus, along with the occlusion of other visual contours from the field of view, did not provide the subjects with a stable reference framework relative to which they could make ASA judgments. In the absence of such a framework, it is likely that the measured ASA shifts are perceptual in nature. This differs from previous investigations of the relationship of ASA shifts to IM employing a rectangular inducer (e.g., Bridgeman
\& Klassen, 1983; Brosgole, 1968), in which a basis for both perceptual and cognitive ASA shifts was provided. ${ }^{1}$

What is the basis of the observed effects of velocity on IM? Although in this study, as well as in others (Bridgeman \& Klassen, 1983; Brosgole, 1968; Post \& Heckmann, 1986), it has been reported that ASA shifts are correlated with IM in the opposite direction, it is unlikely that the ASA shifts played a causal role in the IM observed here. This conclusion is based on an examination of the magnitudes of the two responses. The obtained ASA shifts represent the total displacement at the end of $30 \mathrm{sec}$ and are on the order of a few degrees. Integration of the IM velocity data over the course of the $30-\mathrm{sec}$ stimulus duration demonstrates that the total displacement of the fixated stimulus far exceeds the ASA shift. ${ }^{2}$ This finding is therefore consistent with the conclusions of Mack et al. (1985) and Post and Heckmann (1986).

Of the other subject-relative mechanisms that have been proposed in order to account for IM, we believe that it is unlikely that vection contributed in any significant fashion to the IM measured in the current study, since 2 of the subjects experienced vection consistently, 2 experienced intermittent vection, and 3 did not experience vection at all. Examination of individual results revealed no significant differences in either IM or ASA shifts between those subjects who experienced vection and those who did not. ${ }^{3}$

The findings in the current study are consistent with the hypothesis that IM results from suppression of OKN. That is, the influence of velocity on IM may be related to a similar influence of velocity on $\mathrm{OKN}$ responses (for 
a review, see van Die \& Collewijn, 1982). Specifically, the gain of OKN diminishes at velocities similar to those which produce apparent decreases in IM in the present experiment. At higher velocities, there is less activation of OKN, so there is less need for oppositely directed suppression. This account would also be consistent with the finding that the effect of inducer velocity varies with inducing stimulus size, since Dichgans (1977) has shown that the peak velocity of OKN responses increases in proportion to the horizontal extent of the eliciting stimulus.

What significance is to be made of the finding that ASA shifts are correlated with opposite IM, if the relationship is not causal? As noted in the introduction, ASA shifts, together with OKN and vection, are part of a trilogy of visual-vestibular interactions. Of the three responses, the easiest to measure is the ASA shift. Vection is typically measured by magnitude estimation procedures, wherein subjects compare self-motion sensations to a remembered standard. Some eye-movement recording techniques interfere with the field of view, making their application to IM studies difficult when extended displays are used. In addition, current oculomotor accounts of IM attribute the illusory motion to the suppression of OKN. Therefore, it is necessary to measure optokinetic afternystagmus (OKAN) following termination of the stimulus display, rather than eye movements while observing IM (e.g., Heckmann \& Post, 1988), as OKAN indicates activation of the indirect OKN system (Collewijn, 1985). In comparison with vection and OKAN, it is fairly simple to measure ASA shifts, as has been demonstrated in the present paper. Perhaps the relationship of the ASA shift to IM is ultimately one of utility. That is, ASA shifts may be useful indicators of the presence of either vection or suppressed OKN, regardless of which of these two responses may be causing IM.

\section{REFERENCES}

BeCKLEN, R., WALlaCh, H. (1985). How does speed change affect induced motion? Perception \& Psychophysics, 37, 231-236.

Brecher, G. A., Brecher, M. H., Kommerell, G., Sauter, F. A., \& SELlERBECK, J. (1972). Relation of optical and labyrinthean orientation. Optica Acta, 19, 467-471.

Bridgeman, B., KLASSEN, H. (1983). On the origin of stroboscopic induced motion. Perception \& Psychophysics, 34, 149-154.

Brosgole, L. (1968). Analysis of induced motion. Acta Psychologica, $28,1-44$.

Cohen, B., Matsuo, V., \& Raphan, T. (1977). Quantitative analysis of the velocity characteristics of optokinetic nystagmus and optokinetic afternystagmus. Journal of Physiology (London), 270, 321-344.

CollewIJN, H. (1985). Integration of adaptive changes of optokinetic reflex, pursuit and the vestibulo-ocular reflex. In A. Berthoz \& G. Melvill Jones (Eds.), Adaptive mechanisms in gaze control (pp. 5169). New York: Elsevier.

DichGaNs, J. (1977). Optokinetic nystagmus as dependent on the retinal periphery via the vestibular nucleus. In R. Baker \& A. Berthoz (Eds.), Control of gaze by brain stem neurons (pp. 261-267). Amsterdam: Elsevier/North-Holland.

DichGANS, J., \& BRANDT, T. (1978). Visual-vestibular interaction: Effects on self-motion perception and postural control. In R. Held, H. W.
Leibowitz, \& H.-L. Teuber (Eds.), Handbook of sensory physiology: Vol. 8. Perception (pp. 755-804). Berlin: Springer-Verlag.

Duncker, K. (1929). Über induzierte Bewegung. Psychologische Forschung, 12, 180-259.

HaRRIS, C. S. (1974). Beware of the straight-ahead shift-a nonperceptual change in experiments on adaptation to displaced vision. Perception, 3, 461-476.

Heckmann, T., Howard, I. P. (1989). Induced visual motion: Dissociation of exocentric and egocentric components. Investigative Ophthalmology \& Visual Science, 30(3, Suppl.), 74.

HeckmanN, T., \& Post, R. B. (1988). Induced motion and optokinetic afternystagmus: Parallel response dynamics with prolonged stimulation. Vision Research, 28, 681-694.

HowARD, I. (1989). Perception of egocentric visual direction. In S. Ellis (Ed.), Spatial displays and spatial instruments: A symposium workshop sponsored by the National Aeronautics and Space Administration and the University of California, Berkeley. Hillsdale, NJ: Erlbaum. Johannson, G. (1977). Studies of visual perception of locomotion. Perception, 6, 365-376.

Mack, A., Heuer, F., Fendrich, R., Vilardi, K., \& Chambers, D. (1985). Induced motion and oculomotor capture. Joumal of Experimental Psychology: Human Perception \& Performance, 11, 329-345.

Post, R. B. (1986). Induced motion considered as a visually induced oculogyral illusion. Perception, 15, 131-138.

Post, R. B., Chi, D., Heckmann, T., \& Chaderuian, M. (1989). A reevaluation of the effect of velocity on induced motion. Perception \& Psychophysics, 45, 411-416.

Post, R. B., \& HeckmanN, T. (1986). Induced motion and apparent straight ahead during prolonged stimulation. Perception \& Psychophysics, 40, 263-270.

Post, R. B., \& Leibowitz, H. W. (1985). A revised analysis of the role of efference in motion perception. Perception, 14, 631-643.

REINHARDT-RUTLAND, A. H. (1988). Induced movement in the visual modality: An overview. Psychological Bulletin, 103, 57-71.

Rock, I., Auster, M., SchiffMAN, M., WheEler, D. (1980). Induced movement based on subtraction of motion from the inducing object. Journal of Experimental Psychology: Human Perception \& Performance, 6, 391-403.

van Die, G., \& Collewis, H. (1982). Optokinetic nystagmus in man. Human Neurobiology, 1, 111-119.

Wallach, H., Bacon, J., \& Schulman, P. (1978). Adaptation in motion perception: Alteration of induced motion. Perception \& Psychophysics, 24, 509-514.

WALLACH, H., \& BeCKLEN, R. (1983). An effect of speed on induced motion. Perception \& Psychophysics, 34, 237-242.

\section{NOTES}

1. It should be noted that had the ASA shifts obtained in this experiment been judgmental, the results would not have supported the hypothesis that perceived ASA shifts produce equal and opposite IM, since there would have been no perceived ASA shifts.

2. It might be argued that ASA shifts caused the IM we measured, but that there is an ongoing correction of the ASA back toward the veridical straight-ahead, so that comparison of the cumulative IM does not correspond to the total ASA displacement. Such a hypothesis would, however, posit that some ASA movement produced IM, whereas other ASA movement (the ongoing correction) did not. This would be inconsistent with the hypothesis under examination. Specifically, we were testing whether perceived ASA shifts would produce equal and opposite IM.

3. Heckmann and Howard (1989) have also reported that IM and vection elicited by large inducing stimuli may be dissociated.

(Manuscript received January 12, 1990; revision accepted for publication April 17, 1990.) 